\title{
Lorentz-symmetry violating decays in a medium
}

\author{
José F. Nieves \\ Laboratory of Theoretical Physics \\ Department of Physics, P.O. Box 23343 \\ University of Puerto Rico, Río Piedras, Puerto Rico 00931-3343 \\ Palash B. Pal \\ Saha Institute of Nuclear Physics \\ 1/AF Bidhan-Nagar, Calcutta 700064, India
}

December 2007

\begin{abstract}
Various decay processes, such as the decay of a spin-1 particle into two photons or the gravitational decay of a spin-1/2 fermion, are forbidden in the vacuum by a combination of requirements, including angular momentum conservation, Lorentz invariance and gauge invariance. We show that such processes can occur in a medium, such as a thermal background of particles, even if it is homogeneous and isotropic. We carry out a model-independent analysis of the vertex function for such processes in terms of a set of form factors, and show that the amplitude can be non-zero while remaining consistent with the symmetry principles mentioned above. The results simulate Lorentz symmetry violating effects, although in this case they arise from completely Lorentzinvariant physics.
\end{abstract}

\section{Introduction}

It is well known that the rates of physical process that occur in a medium are modified by the coherent interactions with the background particles. It is also now well known that a medium can induce effects that are not present in the vacuum. For example, in a medium, a chiral fermion can obtain an effective mass [1, 2], or a Majorana fermion can acquire electric and magnetic dipole moments [3, 4, all of which are forbidden in the vacuum. Many other similar effects have been considered in the literature [5]. In general, when the particles propagate through a medium, their properties and interactions are modified such that some processes that are forbidden in the vacuum can be induced by the effects of the medium.

For our purposes, we can divide such processes in two classes. Some processes are forbidden in the vacuum for kinematical reasons. That is, although the off-shell transition 
matrix element is non-zero, the process is forbidden for on-shell particles because of energymomentum conservation. However, in the presence of a medium, the dispersion relations of the particles are modified and those processes can occur. For example, a free electron cannot radiate a photon in the vacuum, but in a medium the dispersion relation of the photon makes the Cerenkov radiation possible. Another example is provided by plasmon decay process $\gamma \rightarrow e^{+} e^{-}$. In the vacuum, it is forbidden due to the masslessness of the photon, but the fact that the photon dispersion relation is modified in the medium makes the process possible. We will not be concerned with this class of processes here.

The other class of processes are those for which the transition matrix element itself is zero in the vacuum. Invariably, whenever that occurs it can be attributed to some conservation laws, which in turn are consequences of the symmetries of the Lagrangian. It is common to refer to such processes as being forbidden. However, in general, a medium is not invariant under the full symmetry group of the Lagrangian. As a consequence, the corresponding transition elements can be non-zero when the effects of the background are included. Thus, for example, the electric and magnetic dipole moments of a Majorana fermion can be non-zero in a medium that is $C P T$-asymmetric [3, 4].

A particular subset of the processes in this second class, which are the focus of this paper, are those which are forbidden in the vacuum by helicity arguments, or angular momentum conservation. Consider, for example, the amplitude for the decay of a spin-0 particle into another spin-0 particle and a photon. In the vacuum, the conservation of angular momentum prevents such processes which, in a more general form, is the statement that electromagnetic interactions cannot take a $J=0$ state to another $J=0$ state. However, the presence of a medium necessarily breaks the Lorentz symmetry and in particular it breaks the isotropy of the three-dimensional space that is responsible for the conservation law that prevents this process from occurring in the vacuum. As a result, if the transition matrix element is calculated taking into account the presence of the background, it will not be zero.

This can be seen in various ways. The medium defines a preferred frame in which all analysis can be performed, namely the frame in which the medium is at rest. If we commit ourselves to this frame and carry out the calculations with respect to this frame, then the expressions for the transition matrix elements are not restricted by Lorentz invariance, and the terms that are absent in the vacuum can appear. Alternatively, if the particle is propagating through the medium, then in the decaying particle's own rest frame the medium is seen as moving with some velocity and this, again, breaks the isotropy of the three-dimensional space.

More generally, we can adopt a completely Lorentz invariant approach by performing the calculation in an arbitrary frame. The medium is then characterized by the temperature and chemical potentials of the background particles, and by the velocity four-vector of its center of mass, $v^{\mu}$. If the particle is at rest in the medium, then $v^{\mu}$ is proportional to the particle's momentum vector $p^{\mu}$ instead of being an independent vector. Therefore, since the amplitude does not depend on any additional vectors apart from the momentum or spin or 
any other vectors that might characterize the initial and final states, the obstructions that apply in the vacuum continue to hold and the amplitude is zero. However, when the particle propagates through the medium, the amplitude in general depends on $v^{\mu}$ in addition to the various vectors characterizing the initial and final particles, which invalidates the symmetry argument based on the isotropy of the three-dimensional space.

In what follows, we adopt the latter point of view. We consider various decay processes which are forbidden in the vacuum by angular momentum conservation and/or helicity arguments. Specifically, we consider the radiative decay of a spin-0 particle into another spin-0 particle, the decay of a spin-1 particle into two photons, the gravitational decay of a spin- 0 particle into another spin- 0 particle and the gravitational decay of a spin- $1 / 2$ particle into another spin- $1 / 2$ particle. In the next sections we review in each case the arguments that show that the amplitude for the process in the vacuum vanishes, and then we demonstrate that the amplitude need not vanish if the process occurs in a medium. In general, the presence of the medium also affects the dispersion relations of the particles appearing in the process. While those effects may be important for the calculations of the rates and specific applications, they are not essential to our arguments and we will ignore them. Although analogous arguments can be given for more complicated processes, we have restricted ourselves to the two-body decay processes mentioned above, which are straightforward to analyze and for which the explicit calculations of the transition amplitudes are simpler. In all our considerations, we assume that the medium is isotropic and that it can be parametrized in the manner indicated above. The last section contains our conclusions.

\section{Radiative decay of a spin-0 particle into another spin-0 particle}

We first consider a process of the form

$$
\phi(p) \rightarrow \phi^{\prime}\left(p^{\prime}\right)+\gamma(q)
$$

where $\phi$ and $\phi^{\prime}$ denote scalar (spin 0) particles, $\gamma$ denotes the photon, and $p, p^{\prime}$ and $q$ denote the corresponding momentum vectors.

We denote by $j^{\mu}$ the off-shell electromagnetic vertex, which is defined such that the on-shell amplitude for the process is given by

$$
\mathscr{M}=\epsilon^{* \mu}(q) j_{\mu}
$$

where

$$
q=p-p^{\prime}
$$


is the photon momentum and $\epsilon^{\mu}(q)$ is the photon polarization vector, which satisfies

$$
q^{\mu} \epsilon_{\mu}(q)=0 .
$$

For on-shell particles, $p, p^{\prime}$ and $q$ satisfy the free-particle dispersion relations,

$$
p^{2}=M^{2}, \quad p^{\prime 2}=M^{\prime 2},
$$

and

$$
q^{2}=0
$$

For electrically neutral scalar particles, the gauge invariance condition implies that

$$
q^{\mu} j_{\mu}=0
$$

for any values of $p^{\mu}$ and $p^{\prime \mu}$. For charged particles, the condition needs to hold only when $j^{\mu}$ is evaluated for $p$ and $p^{\prime}$ satisfying Eq. (2.5).

\subsection{In the vacuum}

In the vacuum, conservation of angular momentum prevents such processes, as mentioned in the Introduction. Let us demonstrate how the result follows, in a manner that will be helpful for analyzing the corresponding case in a material medium.

We take $p^{\mu}$ and $q^{\mu}$ as the independent momentum variables, using Eq. (2.3) to eliminate $p^{\prime}$ in favor of them. Then the vertex function is of the form

$$
j^{\mu}=a_{1} p^{\mu}+a_{2} q^{\mu}
$$

where the coefficients $a_{1,2}$ are scalar functions of $p$ and $q$. In this form, Eq. (2.7) implies that

$$
a_{1} p \cdot q+a_{2} q^{2}=0
$$

Since we are considering the vertex function for a transition amplitude, the fact there is no tree-level electromagnetic coupling between $\phi$ and $\phi^{\prime}$ implies that the vertex function is not singular as $q \rightarrow 0$, and therefore $a$ and $b$ must be of the form

$$
\begin{aligned}
& a_{1}=-q^{2} a_{3}, \\
& a_{2}=(p \cdot q) a_{3},
\end{aligned}
$$

which in turn implies that

$$
j_{\mu}=a_{3}\left[p \cdot q q_{\mu}-q^{2} p_{\mu}\right],
$$

with some undetermined scalar function $a_{3}$. Thus the on-shell amplitude, which is calculated with the conditions given in Eqs. (2.4) and (2.6), is zero. 


\subsection{In a medium}

Let us now consider the same process in a background medium. For our purpose, the crucial difference from the vacuum case is that in this case the vertex function $j^{\mu}$ depends also on the velocity four-vector $v^{\mu}$ of the medium, and therefore its most general form is

$$
j^{\mu}=a_{0} v^{\mu}+a_{1} p^{\mu}+a_{2} q^{\mu}+a_{3} s_{\mu},
$$

where

$$
s_{\mu} \equiv \epsilon_{\mu \alpha \beta \gamma} p^{\alpha} q^{\beta} v^{\gamma} .
$$

The transversality condition of Eq. (2.7) now implies

$$
a_{0} q \cdot v+a_{1} p \cdot q+a_{2} q^{2}=0 .
$$

Solving for $a_{1}$ and substituting the result in Eq. (2.12) yields

$$
j^{\mu}=a_{0}\left[v^{\mu}-\frac{q \cdot v}{p \cdot q} p^{\mu}\right]+a_{2}\left[q_{\mu}-\frac{q^{2}}{p \cdot q} p_{\mu}\right]+a_{3} s_{\mu} .
$$

Using Eqs. (2.4) and (2.6) once more, it follows that the term proportional to $a_{2}$ does not contribute to the on-shell amplitude while the $a_{0,3}$ terms yield

$$
\mathscr{M}=\left(\frac{2 a_{0}}{M^{2}-M^{\prime 2}}\right) F_{\mu \nu}^{*} v^{\mu} p^{\nu}+a_{3} \tilde{F}_{\mu \nu}^{*} v^{\mu} p^{\nu}
$$

where $M$ and $M^{\prime}$ are the masses of the initial and final scalar particles, respectively. In writing Eq. (2.16), we have introduced the notation

$$
F_{\mu \nu}=\epsilon_{\mu} q_{\nu}-q_{\mu} \epsilon_{\nu}
$$

and its dual

$$
\tilde{F}_{\mu \nu}=\frac{1}{2} \epsilon_{\mu \nu \alpha \beta} F^{\alpha \beta}
$$

and used the kinematical relation

$$
2 p \cdot q=M^{2}-M^{\prime 2} .
$$

Thus, the amplitude is not necessarily zero, and therefore the process of Eq. (2.1) can occur in a medium. Written as in Eq. (2.16), it also reveals why this is so. The on-shell amplitude should ultimately contain the factor $F_{\mu \nu}$. In the case of the vacuum, there is no antisymmetric tensor, constructed out of the available independent vectors $p$ and $q$, that gives a non-zero value when contracted with $F_{\mu \nu}$ to produce a scalar amplitude. In the case of the medium, there is one such tensor, as Eq. (2.16) shows. If the particle is at 
rest in the medium, so that $p^{\mu}$ and $v^{\mu}$ are parallel, then the amplitude is zero. This is not unexpected since in that case, in the rest frame of the particle the three-dimensional space is isotropic and the angular momentum conservation argument holds again. But if the particle is moving through the medium, then in its rest frame the three-dimensional space is not isotropic since there the medium is seen moving with some velocity, and therefore the angular momentum conservation argument cannot be applied and the process is not forbidden. In addition, the process can occur for charged as well as electrically neutral scalar particles.

\section{Decay of a spin-1 particle into two photons}

Here consider the process

$$
V(k) \rightarrow \gamma(q)+\gamma\left(q^{\prime}\right),
$$

where $V$ denotes a massive spin-1 particle. We denote the vertex function by $\Gamma_{\mu \alpha \alpha^{\prime}}\left(q, q^{\prime}\right)$ in the vacuum case, or $\Gamma_{\mu \alpha \alpha^{\prime}}\left(q, q^{\prime}, v\right)$ in the presence of the medium, which is such that the amplitude for the process in any case is given by

$$
\mathscr{M}=\epsilon^{* \alpha}(q) \epsilon^{* \alpha^{\prime}}\left(q^{\prime}\right) \Gamma_{\mu \alpha \alpha^{\prime}} \varepsilon^{\mu}(k),
$$

where $\epsilon^{\alpha}(q)$ is the polarization vector for a photon with momentum $q$, while $\varepsilon^{\alpha}(k)$ is the corresponding quantity for the $V$ particle. In analogy with the photon polarization vector, $\varepsilon^{\mu}(k)$ satisfies

$$
k \cdot \varepsilon(k)=0 .
$$

Since all the particles involved in the process are electrically neutral, electromagnetic gauge invariance implies that

$$
\begin{aligned}
q^{\alpha} \Gamma_{\mu \alpha \alpha^{\prime}} & =0 \\
q^{\prime \alpha^{\prime}} \Gamma_{\mu \alpha \alpha^{\prime}} & =0
\end{aligned}
$$

for any values of $q$ and $q^{\prime}$, in the vacuum as well as in the medium. In addition the Bose symmetry under the exchange of the two photons implies that the vertex function satisfies

$$
\begin{aligned}
\Gamma_{\mu \alpha \alpha^{\prime}}\left(q, q^{\prime}\right) & =\Gamma_{\mu \alpha^{\prime} \alpha}\left(q^{\prime}, q\right), \\
\Gamma_{\mu \alpha \alpha^{\prime}}\left(q, q^{\prime}, v\right) & =\Gamma_{\mu \alpha^{\prime} \alpha}\left(q^{\prime}, q, v\right),
\end{aligned}
$$

in the vacuum or in the medium, respectively. In what follows we apply these conditions to the quantity

$$
\Gamma_{\alpha \alpha^{\prime}} \equiv \Gamma_{\mu \alpha \alpha^{\prime}} \varepsilon^{\mu}(k),
$$

taking the $V$-boson on shell, as indicated, while maintaining the photons off-shell. 


\subsection{In the vacuum}

As is well-known, the decay represented in Eq. (3.1) is forbidden in the vacuum by the combination of angular momentum conservation and the Bose symmetry between the two photons, a result known as Yang's theorem [6]. Here we review that result in a way that will help to see how the theorem is evaded in the presence of the medium. The most general form of the vertex function allowed by Lorentz invariance can be read off from an earlier paper [7,

$$
\begin{aligned}
\Gamma_{\mu \alpha \alpha^{\prime}}\left(q, q^{\prime}\right)= & a_{1}\left(q-q^{\prime}\right)_{\mu} \eta_{\alpha \alpha^{\prime}}+\left(a_{2} q_{\alpha}+a_{2}^{\prime} q_{\alpha}^{\prime}\right) \eta_{\mu \alpha^{\prime}}+\left(a_{3} q_{\alpha^{\prime}}+a_{3}^{\prime} q_{\alpha^{\prime}}^{\prime}\right) \eta_{\mu \alpha} \\
& +\left(q-q^{\prime}\right)_{\mu}\left(b_{1} q_{\alpha} q_{\alpha^{\prime}}+b_{1}^{\prime} q_{\alpha}^{\prime} q_{\alpha^{\prime}}^{\prime}+b_{2} q_{\alpha}^{\prime} q_{\alpha^{\prime}}+b_{2}^{\prime} q_{\alpha} q_{\alpha^{\prime}}^{\prime}\right) \\
& +c_{0}\left(q-q^{\prime}\right)_{\mu}\left[q q^{\prime}\right]_{\alpha \alpha^{\prime}}+\left(c_{1} q_{\alpha}+c_{1}^{\prime} q_{\alpha}^{\prime}\right)\left[q q^{\prime}\right]_{\mu \alpha^{\prime}}+\left(c_{2} q_{\alpha^{\prime}}+c_{2}^{\prime} q_{\alpha^{\prime}}^{\prime}\right)\left[q q^{\prime}\right]_{\mu \alpha}
\end{aligned}
$$

where $\eta_{\mu \nu}$ is the Minkowski metric tensor, and we have used the shorthand notation

$$
\left[q q^{\prime}\right]_{\alpha \alpha^{\prime}}=\varepsilon_{\alpha \alpha^{\prime} \beta \gamma} q^{\beta} q^{\prime \gamma}
$$

In writing Eq. (3.8), and in what follows, it should be understood that we are considering the quantity defined in Eq. (3.7), and therefore we omit any term that does not contribute to that quantity. Thus, we have avoided the combination $\left(q+q^{\prime}\right)_{\mu}$ because it vanishes when

it is contracted with $\varepsilon^{\mu}(k)$. Some other possibilities, like $\varepsilon_{\mu \alpha \alpha^{\prime} \beta} q^{\beta}$ and $\varepsilon_{\mu \alpha \alpha^{\prime} \beta} q^{\prime \beta}$, have been omitted since they are not independent [7], as can be seen by contracting the identity

$$
g_{\lambda \rho} \varepsilon_{\alpha \beta \gamma \delta}-g_{\lambda \alpha} \varepsilon_{\rho \beta \gamma \delta}-g_{\lambda \beta} \varepsilon_{\rho \alpha \gamma \delta}-g_{\lambda \gamma} \varepsilon_{\rho \alpha \beta \delta}-g_{\lambda \delta} \varepsilon_{\rho \alpha \beta \gamma}=0
$$

with various combinations of $q$ and $q^{\prime}$.

We now apply the transversality conditions stated in Eqs. (3.4) and (3.5). First of all, they imply

$$
a_{2}=a_{2}^{\prime}=a_{3}=a_{3}^{\prime}=0
$$

and for the other form factors they yield the following relations:

$$
\begin{aligned}
a_{1}+b_{1} q^{2}+b_{2} q \cdot q^{\prime} & =0, \\
a_{1}+b_{1}^{\prime} q^{\prime 2}+b_{2} q \cdot q^{\prime} & =0, \\
b_{1} q \cdot q^{\prime}+b_{2}^{\prime} q^{\prime 2} & =0, \\
b_{1}^{\prime} q \cdot q^{\prime}+b_{2}^{\prime} q^{2} & =0, \\
c_{1} q^{2}+c_{1}^{\prime} q \cdot q^{\prime} & =0, \\
c_{2} q \cdot q^{\prime}+c_{2}^{\prime} q^{\prime 2} & =0 .
\end{aligned}
$$


These can be solved, without introducing singularities, by writing

$$
\begin{aligned}
a_{1} & =B_{1} q^{2} q^{2}-b_{2} q \cdot q^{\prime}, \\
b_{1} & =-B_{1} q^{\prime 2}, \\
b_{1}^{\prime} & =-B_{1} q^{2}, \\
b_{2}^{\prime} & =B_{1} q \cdot q^{\prime}, \\
c_{1} & =C_{1} q \cdot q^{\prime}, \\
c_{1}^{\prime} & =-C_{1} q^{2}, \\
c_{2} & =-C_{2} q^{\prime 2}, \\
c_{2}^{\prime} & =C_{2} q \cdot q^{\prime},
\end{aligned}
$$

and substituting these back in Eq. (3.8) then yields

$$
\begin{aligned}
\Gamma_{\mu \alpha \alpha^{\prime}}\left(q, q^{\prime}\right)= & \left(q-q^{\prime}\right)_{\mu}\left[B_{1} g^{\beta \gamma}\left(q^{2} \eta_{\alpha \beta}-q_{\alpha} q_{\beta}\right)\left(q^{\prime 2} \eta_{\alpha^{\prime} \gamma}-q_{\alpha^{\prime}}^{\prime} q_{\gamma}^{\prime}\right)\right. \\
& \left.+b_{2}\left(q_{\alpha}^{\prime} q_{\alpha^{\prime}}-q \cdot q^{\prime} \eta_{\alpha \alpha^{\prime}}\right)+c_{0}\left[q q^{\prime}\right]_{\alpha \alpha^{\prime}}\right] \\
& +C_{1}\left(q \cdot q^{\prime} q_{\alpha}-q^{2} q_{\alpha}^{\prime}\right)\left[q q^{\prime}\right]_{\mu \alpha^{\prime}}+C_{2}\left(q \cdot q^{\prime} q_{\alpha^{\prime}}^{\prime}-q^{\prime 2} q_{\alpha^{\prime}}\right)\left[q q^{\prime}\right]_{\mu \alpha} .
\end{aligned}
$$

Bose symmetry implies a relationship between $C_{1}$ and $C_{2}$. However, the terms with the coefficients $B_{1}, C_{1}$ and $C_{2}$ do not contribute to the on-shell amplitude given in Eq. (3.2), and we need not consider them further. Regarding the other terms, recall that the form factors are functions only of the scalar invariants $q^{2}$ and $q^{\prime 2}$, since the other invariant, $q \cdot q^{\prime}$, is not independent due to the on-shell condition for the $V$-boson. The Bose symmetry condition, which requires that $b_{2}$ and $c_{0}$ are odd under the interchange of the two photon momenta, is then

$$
b_{2}\left(q^{2}, q^{\prime 2}\right)=-b_{2}\left(q^{\prime 2}, q^{2}\right),
$$

and similarly for $c_{0}$. In particular, this implies that $b_{2}(0,0)=c_{0}(0,0)=0$ for on-shell photons, proving Yang's theorem.

\subsection{In a medium}

In this case, the presence of the vector $v^{\mu}$ allows us to enumerate all the possible terms in a more compact way. This is accomplished by introducing the following vectors,

$$
\begin{aligned}
n_{\lambda} & =\varepsilon_{\lambda \rho \beta \gamma} v^{\rho} q^{\beta} q^{\prime \gamma} \\
t_{\lambda} & =\varepsilon_{\lambda \rho \beta \gamma} n^{\rho} q^{\beta} q^{\prime \gamma}
\end{aligned}
$$


complemented by the following combinations of $q$ and $q^{\prime}$,

$$
\begin{aligned}
r_{\mu} & \equiv q^{2} q_{\mu}^{\prime}-\left(q \cdot q^{\prime}\right) q_{\mu} \\
r_{\mu}^{\prime} & \equiv q^{\prime 2} q_{\mu}-\left(q \cdot q^{\prime}\right) q_{\mu}^{\prime} .
\end{aligned}
$$

Notice that the vector $n_{\mu}$, which is analogous to the vector $s_{\mu}$ defined in Eq. (2.13), as well as $t_{\mu}$ are orthogonal to both $q_{\mu}$ and $q_{\mu}^{\prime}$, while $r_{\mu}$ and $r_{\mu}^{\prime}$ are such that

$$
r \cdot q=r^{\prime} \cdot q^{\prime}=0 .
$$

In addition, the set of vectors

$$
A_{\mu}^{(a)}=\left\{r_{\mu}, r_{\mu}^{\prime}, n_{\mu}, t_{\mu}\right\} \quad(a=1,2,3,4),
$$

are linearly independent in general, and they span the four-dimensional Minkowski space. Therefore, like any rank-3 tensor, $\Gamma_{\mu \alpha \alpha^{\prime}}$ can be expressed in the form

$$
\Gamma_{\mu \alpha \alpha^{\prime}}\left(q, q^{\prime}, v\right)=\sum_{a b c} T_{a b c} A_{\mu}^{(a)} A_{\alpha}^{(b)} A_{\alpha^{\prime}}^{(c)}
$$

but in this case the transversality conditions imply that certain terms in this expression for $\Gamma_{\mu \alpha \alpha^{\prime}}$ are actually absent. The electromagnetic transversality conditions given in Eqs. (3.4) and (3.5) imply that $A_{\alpha}^{(b)}$ and $A_{\alpha^{\prime}}^{(c)}$ can take values only from the subsets $\left\{r_{\alpha}, n_{\alpha}, t_{\alpha}\right\}$ and $\left\{r_{\alpha^{\prime}}^{\prime}, n_{\alpha^{\prime}}, t_{\alpha^{\prime}}\right\}$, respectively. Moreover, if we consider the $V$ to be on-shell (i.e., Eq. (3.7) $)$, then the combination $\left(q+q^{\prime}\right)_{\mu}$ does not contribute and we need to keep only one combination of $r_{\mu}$ and $r_{\mu}^{\prime}$ which we take as $\left(q-q^{\prime}\right)_{\mu}$. We express all this by writing

$$
\varepsilon^{\mu}(k) \Gamma_{\mu \alpha \alpha^{\prime}}\left(q, q^{\prime}, v\right)=\varepsilon^{\mu}(k) \sum_{a b c} T_{a b c}\left\{\left(q-q^{\prime}\right)_{\mu}, t_{\mu}, n_{\mu}\right\}^{(a)}\left\{r_{\alpha}, t_{\alpha}, n_{\alpha}\right\}^{(b)}\left\{r_{\alpha^{\prime}}^{\prime}, t_{\alpha^{\prime}}, n_{\alpha^{\prime}}\right\}^{(c)}
$$

which is the most general form for the vertex function with the $V$ on-shell. For on-shell photons the terms with $r_{\alpha}$ and/or $r_{\alpha^{\prime}}^{\prime}$ give no contribution, and the physical amplitude is

$$
\begin{aligned}
\varepsilon^{\mu}(k) \epsilon^{* \alpha}(q) \epsilon^{* \alpha^{\prime}}\left(q^{\prime}\right) \Gamma_{\mu \alpha \alpha^{\prime}}\left(q, q^{\prime}, v\right)= & \varepsilon^{\mu}(k) \epsilon^{* \alpha}(q) \epsilon^{* \alpha^{\prime}}\left(q^{\prime}\right) \\
& \times \sum_{a b c} \hat{T}_{a b c}\left\{\left(q-q^{\prime}\right)_{\mu}, t_{\mu}, n_{\mu}\right\}^{(a)}\left\{t_{\alpha}, n_{\alpha}\right\}^{(b)}\left\{t_{\alpha^{\prime}}, n_{\alpha^{\prime}}\right\}^{(c)} .
\end{aligned}
$$

This contains 12 form factors, and while Bose symmetry implies certain relations between them, unlike the case in the vacuum the form factors need not vanish as a consequence of this. The reason can be understood by looking at $\hat{T}_{111}$ as an example. Bose symmetry 
implies that it is antisymmetric under the interchange of the two momenta. However, instead of Eq. (3.15), in the present case that condition is

$$
\hat{T}_{111}\left(q^{2}, q^{\prime 2}, q \cdot v, q^{\prime} \cdot v\right)=-\hat{T}_{111}\left(q^{\prime 2}, q^{2}, q^{\prime} \cdot v, q \cdot v\right),
$$

where we have indicated explicitly the dependence on the various independent scalar variables. For on-shell photons this yields the relation

$$
\hat{T}_{111}\left(0,0, q \cdot v, q^{\prime} \cdot v\right)=-\hat{T}_{111}\left(0,0, q^{\prime} \cdot v, q \cdot v\right),
$$

which due to the additional dependence on the variables $q \cdot v$ and $q^{\prime} \cdot v$ does not imply that the on-shell form factor vanishes. In general, therefore, the amplitude for the two-photon decay in a medium does not vanish.

\section{Gravitational decay of a spin-0 particle into another spin-0 particle}

In this section, we consider the process

$$
\phi(p) \rightarrow \phi^{\prime}\left(p^{\prime}\right)+\mathscr{G}(q)
$$

where $\phi$ and $\phi^{\prime}$ denote scalars as before, and $\mathscr{G}$ denotes a graviton. We denote by $t^{\mu \nu}$ the off-shell gravitational vertex, which is symmetric and defined such that the on-shell amplitude for the process is given by

$$
\mathscr{M}=\epsilon^{* \mu \nu}(q) t_{\mu \nu}
$$

where $\epsilon^{\mu \nu}$ is the graviton polarization tensor, which satisfies the relations

$$
\begin{aligned}
\epsilon_{\mu \nu} & =\epsilon_{\nu \mu}, \\
q^{\nu} \epsilon_{\mu \nu}(q) & =0, \\
\eta^{\mu \nu} \epsilon_{\mu \nu} & =0
\end{aligned}
$$

where $\eta_{\mu \nu}$ is the Minkowski metric as before.

Gravitational gauge invariance implies the transversality conditions

$$
q^{\mu} t_{\mu \nu}=q^{\mu} t_{\nu \mu}=0
$$

but in contrast to Eq. (2.7), this is required to hold only for on-shell values of $p$ and $p^{\prime}$. 


\subsection{In the vacuum}

In the vacuum, we can consider $t_{\mu \nu}$ as a function $p^{\mu}$ and $q^{\mu}$ and write it in the form

$$
t_{\mu \nu}=a_{0} \eta_{\mu \nu}+a_{1} p_{\mu} p_{\nu}+a_{2} q_{\mu} q_{\nu}+a_{3}\{p q\}_{\mu \nu},
$$

where the coefficients $a_{i}$ are scalar functions of $p$ and $q$ and

$$
\left\{p_{1} p_{2}\right\}_{\mu \nu} \equiv p_{1 \mu} p_{2 \nu}+p_{2 \mu} p_{1 \nu}
$$

for any two vectors $p_{1,2}$. In this form, Eq. (4.6) then implies the relations

$$
\begin{aligned}
a_{0}+a_{2} q^{2}+a_{3} p \cdot q & =0, \\
a_{1} p \cdot q+a_{3} q^{2} & =0 .
\end{aligned}
$$

Taking into account the fact there is no tree-level gravitational coupling between $\phi$ and $\phi^{\prime}$, which implies that the vertex function is not singular as $q \rightarrow 0$, we solve these relations by setting

$$
\begin{aligned}
& a_{0}=-a_{2} q^{2}-a_{3} p \cdot q, \\
& a_{1} \equiv-a_{4} q^{2}, \\
& a_{3}=a_{4} p \cdot q,
\end{aligned}
$$

which give

$$
t_{\mu \nu}=a_{2}\left(q_{\mu} q_{\nu}-q^{2} \eta_{\mu \nu}\right)+a_{4}\left((p \cdot q)\{p q\}_{\mu \nu}-q^{2} p_{\mu} p_{\nu}-(p \cdot q)^{2} \eta_{\mu \nu}\right) .
$$

The parameters $a_{2,4}$ remain undetermined, but once again, the on-shell graviton amplitude, which is calculated with the conditions given in Eqs. (2.6) and (4.4), is zero.

\subsection{In a medium}

In analogy with the photon case the vertex function in this case depends also on $v^{\mu}$, and we now write

$$
\begin{aligned}
t_{\mu \nu}(p, q, v)= & a_{0} \eta_{\mu \nu}+a_{1} p_{\mu} p_{\nu}+a_{2} q_{\mu} q_{\nu}+a_{3}\{p q\}_{\mu \nu}+a_{4} v_{\mu} v_{\nu}+a_{5}\{p v\}_{\mu \nu}+a_{6}\{q v\}_{\mu \nu} \\
& +b_{1}\{p s\}_{\mu \nu}+b_{2}\{v s\}_{\mu \nu}+b_{3}\{q s\}_{\mu \nu},
\end{aligned}
$$

where $s^{\mu}$ has been defined in Eq. (2.13). The transversality condition yields the following relations,

$$
\begin{aligned}
a_{0}+q^{2} a_{2}+(p \cdot q) a_{3}+(q \cdot v) a_{6} & =0, \\
(p \cdot q) a_{1}+q^{2} a_{3}+(q \cdot v) a_{5} & =0 \\
(q \cdot v) a_{4}+(p \cdot q) a_{5}+q^{2} a_{6} & =0 \\
(p \cdot q) b_{1}+(q \cdot v) b_{2}+q^{2} b_{3} & =0
\end{aligned}
$$


which we use to eliminate $a_{0,1,4}$ and $b_{1}$ in favor of the others, and in this way we arrive at

$$
\begin{aligned}
t_{\mu \nu}(p, q, v)= & a_{2}\left(q_{\mu} q_{\nu}-q^{2} \eta_{\mu \nu}\right)+a_{3}\left[\{p q\}_{\mu \nu}-(p \cdot q) \eta_{\mu \nu}-\left(\frac{q^{2}}{p \cdot q}\right) p_{\mu} p_{\nu}\right] \\
& +a_{5}\left[\{p v\}_{\mu \nu}-\left(\frac{p \cdot q}{q \cdot v}\right) v_{\mu} v_{\nu}-\left(\frac{q \cdot v}{p \cdot q}\right) p_{\mu} p_{\nu}\right] \\
& +a_{6}\left[\{q v\}_{\mu \nu}-(q \cdot v) \eta_{\mu \nu}-\left(\frac{q^{2}}{q \cdot v}\right) v_{\mu} v_{\nu}\right] \\
& +b_{2}\left[\{v s\}_{\mu \nu}-\left(\frac{q \cdot v}{p \cdot q}\right)\{p s\}_{\mu \nu}\right]+b_{3}\left[\{q s\}_{\mu \nu}-\left(\frac{q^{2}}{p \cdot q}\right)\{p s\}_{\mu \nu}\right]
\end{aligned}
$$

For an on-shell graviton, the terms proportional to $a_{2,3,6}$ and $b_{3}$ vanish, but the terms with the coefficient $a_{5}$ and $b_{2}$ give a non-vanishing contribution to the amplitude,

$$
\begin{aligned}
\mathscr{M}= & a_{5} \epsilon^{* \mu \nu}(q)\left[\{p v\}_{\mu \nu}-\left(\frac{p \cdot q}{q \cdot v}\right) v_{\mu} v_{\nu}-\left(\frac{q \cdot v}{p \cdot q}\right) p_{\mu} p_{\nu}\right] \\
& +b_{2} \epsilon^{* \mu \nu}(q)\left[\{v s\}_{\mu \nu}-\left(\frac{q \cdot v}{p \cdot q}\right)\{p s\}_{\mu \nu}\right] .
\end{aligned}
$$

\section{Gravitational decay of a spin-1/2 particle into another spin-1/2 particle}

We denote by $\Gamma_{\mu \nu}(p, q)$ the off-shell gravitational vertex function, which is defined such that the amplitude for the process

$$
f(p) \rightarrow f^{\prime}\left(p^{\prime}\right)+\mathscr{G}(q)
$$

is given by

$$
\mathscr{M}=\epsilon^{* \mu \nu} \bar{u}_{f^{\prime}}\left(p^{\prime}\right) \Gamma_{\mu \nu} u_{f}(p) .
$$

The vertex function $\Gamma_{\mu \nu}$ is symmetric in its indices, and gravitational gauge invariance implies that it satisfies the transversality conditions

$$
q^{\mu} \bar{u}_{f^{\prime}}\left(p^{\prime}\right) \Gamma_{\mu \nu} u_{f}(p)=q^{\mu} \bar{u}_{f^{\prime}}\left(p^{\prime}\right) \Gamma_{\nu \mu} u_{f}(p)=0 .
$$

In order to write down the general form of the matrix element $\bar{u}_{f^{\prime}}\left(p^{\prime}\right) \Gamma_{\mu \nu} u_{f}(p)$ we introduce its tensor and pseudotensor components by writing

$$
\bar{u}_{f^{\prime}}\left(p^{\prime}\right) \Gamma_{\mu \nu} u_{f}(p)=\bar{u}_{f^{\prime}}\left(p^{\prime}\right)\left[\Gamma_{\mu \nu}^{(T)}+\Gamma_{\mu \nu}^{(P)} \gamma_{5}\right] u_{f}(p) .
$$

The transversality condition then implies that

$$
q^{\nu} \bar{u}_{f^{\prime}}\left(p^{\prime}\right) \Gamma_{\mu \nu}^{(i)} u_{f}(p)=0,
$$

for $i=T, P$ independently. 


\subsection{In the vacuum}

With the understanding that the expression $\Gamma_{\mu \nu}^{(T)}+\Gamma_{\mu \nu}^{(P)} \gamma_{5}$ is sandwiched between the spinors, the most general form of each of the components $\Gamma_{\mu \nu}^{(T, P)}$ is

$$
\Gamma_{\mu \nu}^{(i)}=a_{0}^{(i)} \eta_{\mu \nu}+a_{1}^{(i)} p_{\mu} p_{\nu}+a_{2}^{(i)} q_{\mu} q_{\nu}+a_{3}^{(i)}\{p q\}_{\mu \nu}+a_{4}^{(i)}\{p \gamma\}_{\mu \nu}+a_{5}^{(i)}\{q \gamma\}_{\mu \nu},
$$

where we have used the Dirac equation for the spinors to reduce the terms that contain factors of $\not p$ or $\not$. In addition, the terms involving $\epsilon_{\mu \alpha \beta \gamma} p^{\alpha} q^{\beta} \gamma^{\gamma}$ can be reduced to the ones above by using the identity

$$
\gamma_{\alpha} \gamma_{\beta} \gamma_{\gamma}=\left(\eta_{\mu \alpha} \eta_{\beta \gamma}-\eta_{\mu \beta} \eta_{\alpha \gamma}+\eta_{\mu \gamma} \eta_{\alpha \beta}\right) \gamma^{\mu}+i \epsilon_{\alpha \beta \gamma \mu} \gamma^{\mu} \gamma_{5}
$$

together with the Dirac equation.

Let us consider the tensor $(T)$ component first. Using the Dirac equation for the spinors to reduce the factors of $\not$ that appear, the transversality conditions imply

$$
\begin{aligned}
a_{0}^{(T)}+q^{2} a_{2}^{(T)}+(p \cdot q) a_{3}^{(T)}+\left(m-m^{\prime}\right) a_{5}^{(T)} & =0, \\
(p \cdot q) a_{1}^{(T)}+q^{2} a_{3}^{(T)}+\left(m-m^{\prime}\right) a_{4}^{(T)} & =0, \\
(p \cdot q) a_{4}^{(T)}+q^{2} a_{5}^{(T)} & =0,
\end{aligned}
$$

To satisfy the last relation we set

$$
\begin{aligned}
& a_{4}^{(T)}=-q^{2} a_{6}^{(T)}, \\
& a_{5}^{(T)}=(p \cdot q) a_{6}^{(T)},
\end{aligned}
$$

and then we use the first and second relations to solve for $a_{0}^{(T)}$ and $a_{1}^{(T)}$, respectively, obtaining

$$
\begin{aligned}
& a_{0}^{(T)}=-q^{2} a_{2}^{(T)}-(p \cdot q) a_{3}^{(T)}-\left(m-m^{\prime}\right)(p \cdot q) a_{6}^{(T)} \\
& a_{1}^{(T)}=\frac{q^{2}}{p \cdot q}\left(\left(m-m^{\prime}\right) a_{6}^{(T)}-a_{3}^{(T)}\right) .
\end{aligned}
$$

For the $(P)$ component the results are similar, the only difference being that in the formulas analogous to Eq. (5.10), instead of $m-m^{\prime}$ the factor $-m-m^{\prime}$ appears. Thus, for either component,

$$
\begin{aligned}
\Gamma_{\mu \nu}^{(i)}= & a_{2}^{(i)}\left(q_{\mu} q_{\nu}-q^{2} \eta_{\mu \nu}\right)+a_{3}^{(i)}\left[\{p q\}_{\mu \nu}-\frac{q^{2}}{p \cdot q} p_{\mu} p_{\nu}-(p \cdot q) \eta_{\mu \nu}\right] \\
& +a_{6}^{(i)}\left\{q^{2}\left[\frac{\not}{p \cdot q} p_{\mu} p_{\nu}-\{p \gamma\}_{\mu \nu}\right]+(p \cdot q)\left[\{q \gamma\}_{\mu \nu}-\not q \eta_{\mu \nu}\right]\right\},
\end{aligned}
$$

where we have used the Dirac equation once again to rewrite the factors of $m \pm m^{\prime}$ in terms $\not$. The relations in Eqs. (4.4) and (4.5) then imply that, for an on-shell graviton, the amplitude is zero. 


\subsection{In a medium}

In this case, the terms involving the factors $\psi, \sigma_{\alpha \beta} v^{\beta}$ and $\epsilon_{\mu \alpha \beta \gamma} \ell^{\alpha} v^{\beta} \gamma^{\gamma}$ (where $\ell=p, q$ ), must be taken into account. Therefore we write, for $i=T, P$ as before,

$$
\begin{aligned}
\Gamma_{\mu \nu}^{(i)}= & \eta_{\mu \nu}\left(a_{0}^{(i)}+b_{0}^{(i)} \psi\right)+p_{\mu} p_{\nu}\left(a_{1}^{(i)}+b_{1}^{(i)} \not\right)+q_{\mu} q_{\nu}\left(a_{2}^{(i)}+b_{2}^{(i)} \psi\right)+v_{\mu} v_{\nu}\left(a_{3}^{(i)}+b_{3}^{(i)} \psi\right) \\
& +\{p q\}_{\mu \nu}\left(a_{4}^{(i)}+b_{4}^{(i)} \not\right)+\{p v\}_{\mu \nu}\left(a_{5}^{(i)}+b_{5}^{(i)} \not\right)+\{q v\}_{\mu \nu}\left(a_{6}^{(i)}+b_{6}^{(i)} \not\right) \\
& +\{p \gamma\}_{\mu \nu}\left(a_{7}^{(i)}+b_{7}^{(i)} \not\right)+\{q \gamma\}_{\mu \nu}\left(a_{8}^{(i)}+b_{8}^{(i)} \not\right)+\{v \gamma\}_{\mu \nu}\left(a_{9}^{(i)}+b_{9}^{(i)} \not\right)
\end{aligned}
$$

where we have chosen to write the terms with two $\gamma$ matrices in terms of $\gamma_{\mu} \psi$ rather than $\sigma_{\alpha \beta} v^{\beta}$. In addition, the terms with the epsilon symbol mentioned above have been omitted since they reduce to those that are included here by using the identity Eq. (5.7) and the Dirac equation.

As in the vacuum case, the transversality condition must be satisfied for $\Gamma_{\mu \nu}^{(T, P)}$ separately, and furthermore, within each group, the terms with and without $\psi$ must vanish separately as well. In this way we obtain the following relations,

$$
\begin{aligned}
a_{0}^{(i)} & =-q^{2} a_{2}^{(i)}-p \cdot q a_{4}^{(i)}-q \cdot v a_{6}^{(i)}-\not q a_{8}^{(i)} \\
a_{1}^{(i)} & =\frac{-1}{p \cdot q}\left[q^{2} a_{4}^{(i)}+q \cdot v a_{5}^{(i)}-\frac{\not 1}{p \cdot q}\left(q^{2} a_{8}^{(i)}+q \cdot v a_{9}^{(i)}\right)\right], \\
a_{3}^{(i)} & =\frac{-1}{v \cdot q}\left[p \cdot q a_{5}^{(i)}+q^{2} a_{6}^{(i)}+\not q a_{9}^{(i)}\right] \\
a_{7}^{(i)} & =\frac{-1}{p \cdot q}\left[q^{2} a_{8}^{(i)}+q \cdot v a_{9}^{(i)}\right]
\end{aligned}
$$

with analogous relations for the set of coefficients $b_{j}^{(i)}$, and we obtain the final form

$$
\begin{aligned}
\Gamma_{\mu \nu}^{(i)}= & \left(-q^{2} \eta_{\mu \nu}+q_{\mu} q_{\nu}\right)\left(a_{2}^{(i)}+b_{2}^{(i)} \not\right) \\
& +\left[-p \cdot q \eta_{\mu \nu}-\frac{q^{2}}{p \cdot q} p_{\mu} p_{\nu}+\{p q\}_{\mu \nu}\right]\left(a_{4}^{(i)}+b_{4}^{(i)} \psi\right) \\
& +\left[-\frac{q \cdot v}{p \cdot q} p_{\mu} p_{\nu}-\frac{p \cdot q}{q \cdot v} v_{\mu} v_{\nu}+\{p v\}_{\mu \nu}\right]\left(a_{5}^{(i)}+b_{5}^{(i)} \psi\right) \\
& +\left[-q \cdot v \eta_{\mu \nu}-\frac{q^{2}}{q \cdot v} v_{\mu} v_{\nu}+\{q v\}_{\mu \nu}\right]\left(a_{6}^{(i)}+b_{6}^{(i)} \psi\right) \\
& +\left[-\not q \eta_{\mu \nu}+\frac{q^{2}}{(p \cdot q)^{2}} \not p_{\mu} p_{\nu}-\frac{q^{2}}{p \cdot q}\{p \gamma\}_{\mu \nu}+\{q \gamma\}_{\mu \nu}\right]\left(a_{8}^{(i)}+b_{8}^{(i)} \psi\right) \\
& +\left[\frac{q \cdot v}{(p \cdot q)^{2}} \not p p_{\mu} p_{\nu}-\frac{\not d}{q \cdot v} v_{\mu} v_{\nu}-\frac{q \cdot v}{p \cdot q}\{p \gamma\}_{\mu \nu}+\{v \gamma\}_{\mu \nu}\right]\left(a_{9}^{(i)}+b_{9}^{(i)} \psi\right) .
\end{aligned}
$$


Some of the terms in Eq. (5.14) vanish for an on-shell graviton. However, the terms proportional to the coefficients $a_{5,9}^{(i)}$ and $b_{5,9}^{(i)}$ do not vanish and therefore the physical amplitude can be non-zero in a medium.

\section{Conclusions}

We have considered various decay processes which are known to be forbidden in the vacuum by a combination of requirements such as angular momentum conservation, Lorentz invariance or gauge invariance. As we showed, such processes can occur in a medium, such as a thermal background of particles, despite the fact that the medium may be homogeneous and isotropic. To be precise, we carried out a model-independent analysis of the vertex function for such processes in terms of a set of form factors, and showed that the amplitude can be non-zero while remaining consistent with the symmetry principles mentioned above. The results simulate Lorentz symmetry violating effects, although in this case they arise from completely Lorentz-invariant physics.

The idea that the Lorentz symmetry is not exact, and the possible physical consequences of this, has been of interest and an active field of research in the recent times [8, 9, 10, 11, 12]. The model-independent parametrization performed in the present work is useful in these contexts as well. Firstly, it can help to discriminate between the effects produced by genuine Lorentz invariance violation at a fundamental level, from similar effects that may arise even if it is not really violated. Secondly, the calculation of the form factors that we have defined require the specification of the background for the physical situation at hand, but otherwise does not depend on any new physics beyond the standard model. Therefore, the results of such calculations can be used as benchmark values with which to compare the results of similar calculations in the context of models of genuine Lorentz symmetry violation.

\section{References}

[1] L. Wolfenstein, Phys. Rev. D 17, 2369 (1978).

[2] H. A. Weldon, Phys. Rev. D 26, 2789 (1982).

[3] J. F. Nieves and P. B. Pal, Phys. Rev. D 40, 1693 (1989).

[4] V. B. Semikoz and Y. A. Smorodinsky, Sov. Phys. JETP 68, 20 (1989) [Zh. Eksp. Teor. Fiz. 95, 35 (1989)].

[5] See, e.g., J. C. D’Olivo, J. F. Nieves and P. B. Pal, Phys. Rev. Lett. 64, 1088 (1990); R. F. Sawyer, Phys. Rev. D 46, 1180 (1992); C. Giunti, C. W. Kim, U. W. Lee and W. P. Lam, Phys. Rev. D 45, 1557 (1992); J. F. Nieves and P. B. Pal, Phys. Rev. D 49, 
1398 (1994) arXiv:hep-ph/9305308. J. C. D'Olivo, J. F. Nieves and P. B. Pal, Phys. Lett. B 365, 178 (1996) arXiv:hep-ph/9509415; J. F. Nieves and P. B. Pal, Phys. Rev. D 56, 365 (1997) arXiv:hep-ph/9702283.

[6] C. N. Yang, Phys. Rev. 77, 242 (1950).

[7] J. F. Nieves and P. B. Pal, Phys. Rev. D 55, 3118 (1997) arXiv:hep-ph/9611431.

[8] S. M. Carroll, G. B. Field and R. Jackiw, Phys. Rev. D 41, 1231 (1990).

[9] S. R. Coleman and S. L. Glashow, Phys. Rev. D 59, 116008 (1999) arXiv:hep-ph/9812418.

[10] R. Jackiw and V. A. Kostelecky, Phys. Rev. Lett. 82, 3572 (1999) arXiv:hep-ph/9901358.

[11] G. L. Fogli, E. Lisi, A. Marrone and G. Scioscia, Phys. Rev. D 60, 053006 (1999) arXiv:hep-ph/9904248.

[12] For reviews and further references see, for example, V. A. Kostelecký, Third meeting on CPT and Lorentz Symmetry (World Scientific, Singapore, 2005); G. M. Shore, Nucl. Phys. B 717, 86 (2005). D. Mattingly, Living Rev. Rel. 8, 5 (2005); T. Jacobson, S. Liberati, and D. Mattingly, Ann. Phys. 321, 150 (2006). 